Provided for non-commercial research and education use. Not for reproduction, distribution or commercial use.

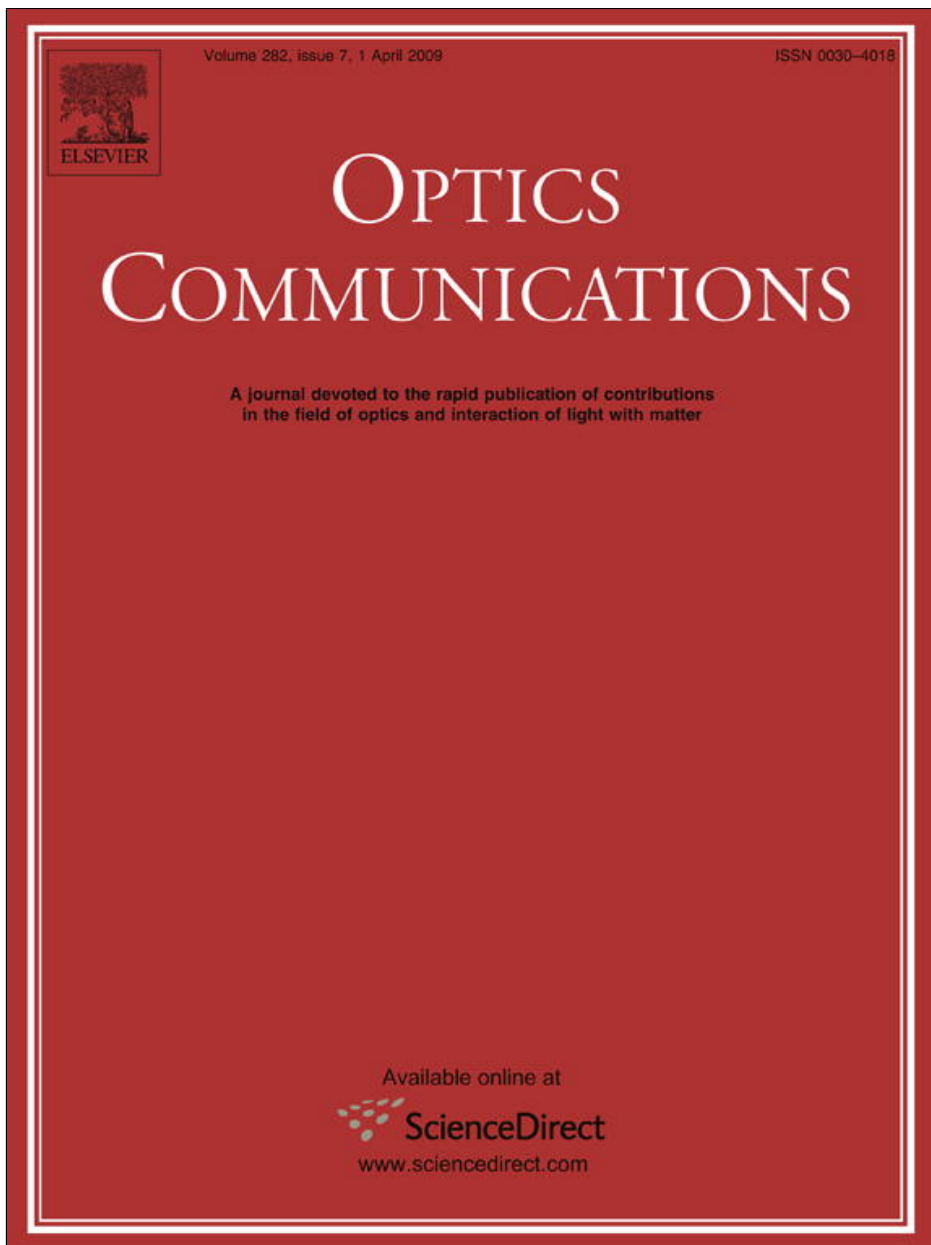

This article appeared in a journal published by Elsevier. The attached copy is furnished to the author for internal non-commercial research and education use, including for instruction at the authors institution and sharing with colleagues.

Other uses, including reproduction and distribution, or selling or licensing copies, or posting to personal, institutional or third party websites are prohibited.

In most cases authors are permitted to post their version of the article (e.g. in Word or Tex form) to their personal website or institutional repository. Authors requiring further information regarding Elsevier's archiving and manuscript policies are encouraged to visit:

http://www.elsevier.com/copyright 


\title{
Complex Padé approximant operators for wide-angle beam propagation
}

\author{
Khai Q. Le \\ Department of Information Technology, Ghent University-IMEC, St-Pietersnieuwstraat 41, B-9000 Ghent, Belgium
}

\section{A R T I C L E I N F O}

\section{Article history:}

Received 16 October 2008

Received in revised form 5 December 2008

Accepted 8 December 2008

\begin{abstract}
A B S T R A C T
The conventional rational Hadley $(m, n)$ approximant of wide-angle beam propagator based on real Padé approximant operators incorrectly propagates the evanescent modes. In order to overcome this problem, two complex Padé approximants of wide-angle beam propagator are presented in this paper. The complex propagators of the first approach are obtained by using the same recurrence formula from the scalar Helmholtz equation of the conventional approximant method with a different initial value while those of the second method derived from $\operatorname{Hadley}(m, n)$ approximant of a square-root operator that has been rotated in the complex plane. These resulting approaches allow more accurate approximations to the Helmholtz equation than the well-known real Padé approximant. Furthermore, our proposed complex Padé approximant operators give the evanescent modes the desired damping.
\end{abstract}

(c) 2008 Elsevier B.V. All rights reserved.

\section{Introduction}

The wide-angle (WA) beam propagation method (BPM) has become one of the most widely used techniques for the study of optical waveguide devices [1,2]. Different treatments of WA-BPM based on the slowly varying envelope approximation have been developed, including the rational approximants of the square root operator [3], the one-way propagator [4], the exponential of the square root operator [5], and the real Padé approximant operator [6] (referred to as $\operatorname{Hadley}(m, n)$ approximant thereafter in this work), for rectangular coordinates as well as an oblique coordinate system [7]. The Padé-approximant-based WA-BPM is one of the most commonly used techniques for modeling optical waveguide structures. It is a nonlinear expression in the form of a rational function $\frac{N(m)}{D(n)}$, a ratio of two polynomials in operator $P$ [6], where $\mathrm{m}$ and $\mathrm{n}$ are the highest degree of $P$ in the polynomials $N$ and $D$, respectively. They have also been used in connection with a number of physical problems.

However, as the denominator $D(n)$ of the rational function of $\operatorname{Hadley}(m, n)$ approximant gradually approaches zero, its absolute values are thus indefinite. It physically causes the fact that the real Padé approximant or $\operatorname{Hadley}(m, n)$ propagators incorrectly propagate the evanescent modes leading to additional errors to the final solution. Furthermore, these waves can cause serious instability problems when implementing WA-BPM based on real Padé approximants. To circumvent this problem we propose two approaches resulting in complex Padé approximant operators that give the evanescent modes the desired damping and thus allow more accurate approximations to the Helmholtz equation than real Padé approximants.

E-mail address: khai.le@intec.ugent.be

\section{Formulation}

\subsection{Real Padé approximant operators}

The scalar Helmholtz equation obtained by using the slowly varying envelope approximation is given by [6]

$\frac{\partial H}{\partial z}-\frac{i}{2 k} \frac{\partial^{2} H}{\partial z^{2}}=\frac{i P}{2 k} H$

where $P=\nabla_{\perp}^{2}+k_{0}^{2}\left(n^{2}-n_{\text {ref }}^{2}\right)=\frac{\partial^{2}}{\partial x^{2}}+\frac{\partial^{2}}{\partial y^{2}}+k_{0}^{2}\left(n^{2}-n_{\text {ref }}^{2}\right)$ with $k=$ $k_{0} n_{\text {ref }}, n_{\text {ref }}$ being the reference refractive index, for which the refractive index of the substrate or cladding is usually used [8], $k_{0}$ being the vacuum wavevector, $H(x, y, z) \exp (i \omega t)$ being the field component with angular frequency $\omega$.

We may formally rewrite Eq. (1) in the form

$\frac{\partial H}{\partial z}=i \frac{\frac{P}{2 k}}{1-\frac{i}{2 k} \frac{\partial}{\partial z}} H$.

Eq. (2) suggests the recurrence relation

$\left.\frac{\partial}{\partial z}\right|_{n+1}=i \frac{\frac{P}{2 k}}{1-\left.\frac{i}{2 k} \frac{\partial}{\partial z}\right|_{n}}$.

Hadley [6] proposed the rational approximation of WA beam propagation using real Padé approximant operators with initial value of $\left.\frac{\partial}{\partial z}\right|_{0}=0$.

This gives us the well-known Padé $(m, n)$ approximant-based WA beam propagation formula as follows:

$\frac{\partial H}{\partial z} \approx i k \frac{N(m)}{D(n)} H$.

where $N(m)$ and $D(n)$ are polynomials in $X=\frac{P}{k^{2}}$. 
If Eq. (4) is compared with a formal solution of Eq. (1) written in the well-known form [6]

$\frac{\partial H}{\partial z}=i\left(\sqrt{P+k^{2}}-k\right) H=i k(\sqrt{1+X}-1) H$,

we obtained the approximation formula

$\sqrt{1+X}-1 \approx \frac{N(m)}{D(n)}$.

Since the operator $X$ has a real spectrum, it is useful to consider the approximation of $\sqrt{1+X}-1$ by the Padé approximant propagation operator. Fig. 1 shows the absolute values of $\sqrt{1+X}-1$ and the first order real Padé(1,1) approximant operator or Hadley $(1,1)$ with respect to $X$.

However, as the denominator of the rational approximation of WA beam propagation using $\operatorname{Hadley}(m, n)$ approximant gradually approaches zero, its absolute value is thus indefinite as clearly seen in Fig. 2. To circumvent this problem we propose here two approaches that lead to complex Padé approximant operators.

\subsection{Complex Padé approximant operators}

\subsubsection{Modified Padé approximant operators}

By multiplying both sides of Eq. (2) with $-\frac{i}{k}$, we obtain

$-\frac{i}{k} \frac{\partial}{\partial z}=\frac{\frac{P}{k^{2}}}{2-\frac{i}{k} \frac{\partial}{\partial z}}$

We may rewrite Eq. (7) as follows

$f(X)=\frac{X}{2+f(X)}$.

where $f(X)=-\frac{i}{k} \frac{\partial}{\partial z}$.

Eq. (8) suggests the recurrence relation

$f_{n+1}(X)=\frac{X}{2+f_{n}(X)} \quad$ for $n=0,1,2 \ldots$

Lu [9] has proved that Eq. (9) can provide a good approximation to $\sqrt{1+X}-1$ with the initial value of

$f_{0}(X)=i \beta \quad$ where $\beta>0$.

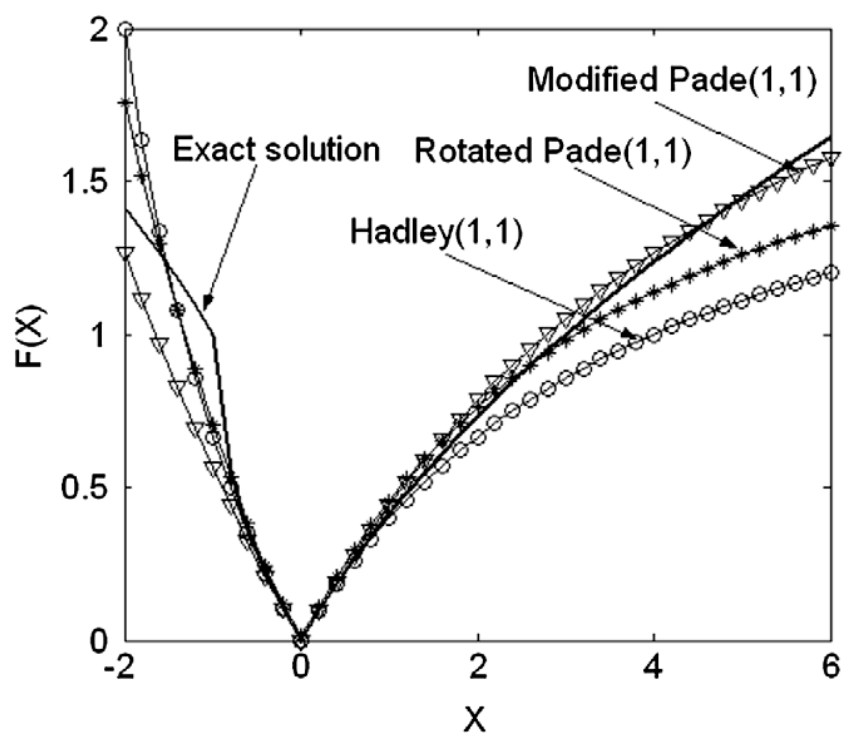

Fig. 1. The absolute values of $F(X)=\sqrt{1+X}-1$ (solid line), the first-order real (solid line with circles), complex modified (solid line with triangular) and rotated (solid line with stars) Padé(1,1) approximant of $\sqrt{1+X}-1$.



Fig. 2. The absolute values of $F(X)=\sqrt{1+X}-1$ (solid line), the first-order real (solid line with circles), complex modified (solid line with down triangles) and rotated (solid line with stars) Padé(1,1) approximant of $\sqrt{1+X}-1$.

Table 1

Most useful low-order Padé approximants of $\sqrt{1+X}-1$ in terms of the operator $X$.

\begin{tabular}{|c|c|}
\hline Order & Expression \\
\hline$(1,0)$ & $\frac{X}{2\left(1+\frac{i \beta}{2}\right)}$ \\
\hline$(1,1)$ & $\frac{\frac{X}{2}}{1+\frac{X}{4\left(1+\frac{j \beta}{2}\right)}}$ \\
\hline$(2,2)$ & $\frac{\frac{X}{2}+\left(1+\frac{1}{\left.1+\frac{j}{2}\right)} \frac{X^{2}}{8}\right.}{1+\left(2+\frac{1}{2}\right) \frac{\frac{X}{4}}{4}+\frac{X^{2}}{2}+\frac{X^{2}}{16\left(1+\frac{j \beta}{2}\right)}}$ \\
\hline$(3,3)$ & 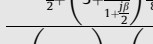 \\
\hline
\end{tabular}

Subsequently, our modified Padé approximant operators are obtained from the same recurrence formula (3) with a different initial value of $\left.\frac{\partial}{\partial z}\right|_{0}=-k \beta$. The most useful low-order modified Padé approximant operators are shown in Table 1.

\subsubsection{Rotated Padé approximant operators}

Several years ago, Milinazzo [3] proposed the rotation of the square-root operator in the complex plane to address the evanescent waves as follows:

$\sqrt{1+X}-1=\sqrt{(1+X) e^{-j \theta} e^{j \theta}}-1=e^{j \theta / 2} \sqrt{1+Z}-1$

where $Z=(1+X) e^{-j \theta}-1$. In addition, from Eq. (6) we obtain the $\operatorname{Hadley}(m, n)$ approximant of the square-root operator with respect to $X$

$\left.\sqrt{1+X} \approx \frac{N(m)}{D(n)}\right|_{X}+1$

Therefore, by employing the $\operatorname{Hadley}(m, n)$ approximant of the square-root operator with respect to $Z$ and inserting it into Eq. (11), we obtain rotated Padé approximant operators as follows:

$\sqrt{1+X}-1=e^{j \theta / 2} \sqrt{1+Z}-1 \approx e^{j \theta / 2}\left(\left.\frac{N(m)}{D(n)}\right|_{Z}+1\right)-1$. 


\section{Numerical results}

In this section, we compare two complex Padé approximants to the exact square-root operator $\sqrt{1+X}-1$. The complex Padé approximants to the propagator are obtained using the first method based on the modified Padé approximant with $\beta=2$ [9] while those of the second method based on the rotated Padé approximant propagator are obtained using a rotation angle of $\theta=\frac{\pi}{4}$ [3]. Fig. 1 also shows the absolute values of the first order complex Padé approximant operators using these methods with respect to $\mathrm{X}$. It is obvious that these complex Padé approximant operators can provide better approximations to the exact square-root operator $\sqrt{1+X}-1$ than those of the real Padé approximant. Furthermore, the real rational Padé approximant-based propagators incorrectly propagates the evanescent modes as their denominator gradually approaches zero while the complex Padé approximants

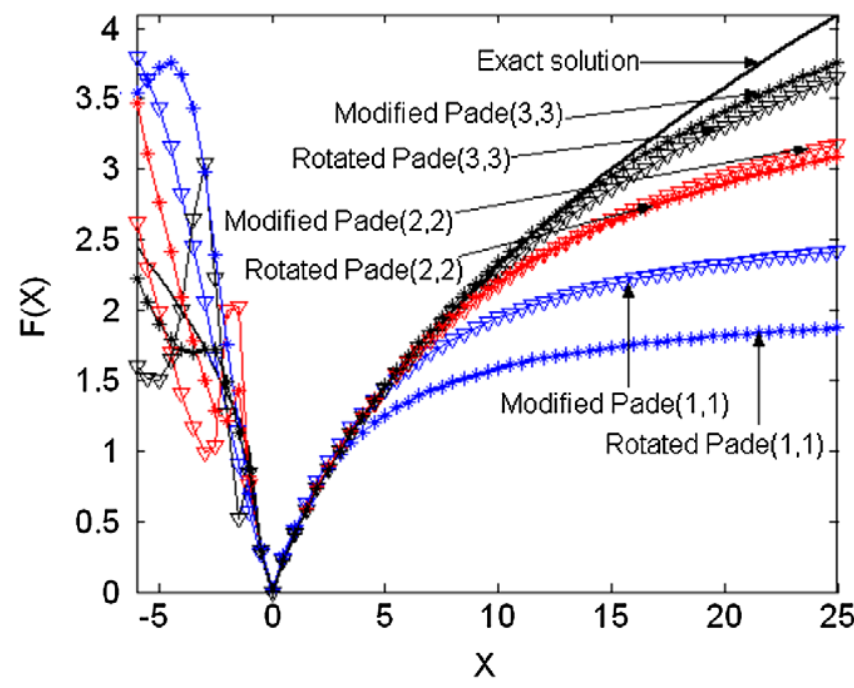

Fig. 3. The absolute values of $F(X)=\sqrt{1+X}-1$, the most useful low-order real Padé approximant and complex modified and rotated Padé approximants of $\sqrt{1+X}-1$.

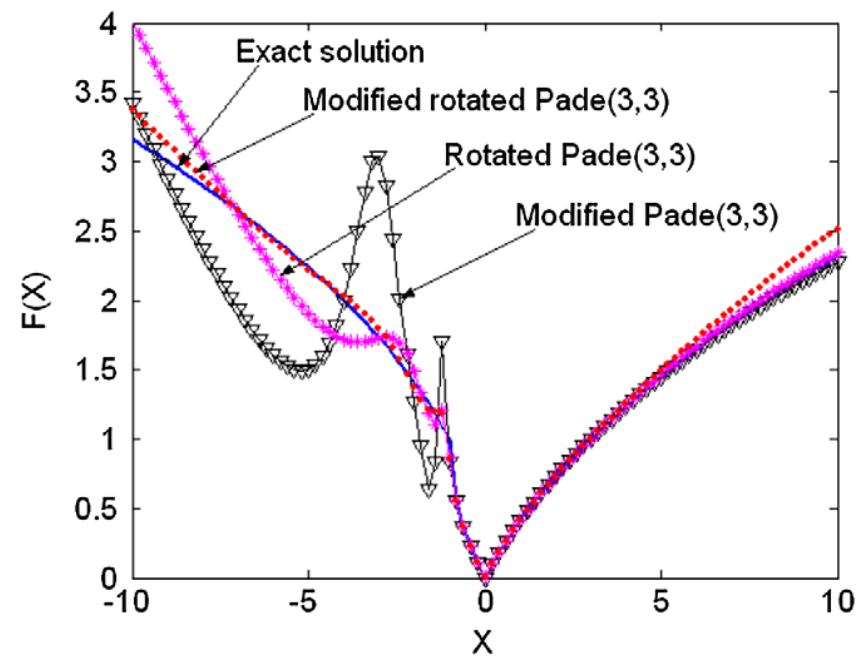

Fig. 4. The absolute values of $F(X)=\sqrt{1+X}-1$ (solid line), the fifth-order rea (solid line with circles) Padé(3,3) approximant and complex modified (solid line with down triangles), rotated (solid line with stars) and modified rotated (red doted lines) Padé(3,3) approximants of $\sqrt{1+X}-1$. give the evanescent mode the desired damping as clearly seen in Fig. 2.

In Fig. 3 we show that for low-order cases, the modified Padé approximants allow more accurate resolution of the propagating modes than those of the rotated Padé approximants. However for high-order cases, the rotated Padé approximants can provide better description of the evanescent waves as clearly seen in Fig. 4 and, in particular, allow more accurate approximations to the Helmholtz equation in the propagating mode regime than those of the modified Padé approximants. Further, the optimal procedure for constructing modified Padé approximants to the rotated square-root operator mentioned here as modified rotated Padé approximants can offer improved accuracy in the evanescent region of the spectrum. However, this optimal approach is somewhat less accurate than the two previous approaches in the propagating mode regime as also shown in Fig. 4. Consequently, the choice of an optimal procedure is highly problem-dependent.

For an implementation of WA-BPM based on higher-order Padé $(m, n)$ approximants, various treatments have been developed to relax the computational efforts including the multistep method [10], the splitting method of the wave equation [11] or the shifting technique of the simulation window to reduce the dimension of the numerical equation [12]... In general, these methods are usually developed based on the traditional direct matrix inversion or the iterative method whereby the propagation equation can be cast in terms of a Helmholtz equation with source term [13]. However, for certain problems, iterative methods can be instable for dealing with WA-BPM based on real Padé $(m, n)$ approximants whereas those of complex Padé $(m, n)$ approximants-based method can reduce the instability problems since evanescent waves are always damped during propagation.

\section{Conclusion}

The complex Padé approximants of WA beam propagation operator have been presented. The resulting approaches allow more accurate resolutions of the propagating modes than those of the real Padé approximant-based approach. Furthermore, these approaches ensure the evanescent waves always decay during propagation. Therefore, these complex approximants allow more accurate approximations to the Helmholtz equation than those of the well-known real Padé approximants.

\section{Acknowledgements}

The author would like to thank Prof. Peter Bienstman (Department of Information Technology, Ghent University) for invaluable discussions on this work.

\section{References}

[1] R. Scarmozzino, A. Gopinath, R. Pregla, S. Helfert, IEEE J. Sel. Top. Quantum Electron. 6 (2000) 150.

[2] Y.Y. Lu, Commun. Comput. Phys. 1 (2006) 1056

[3] F.A. Milinazzo, C.A. Zala, G.H. Brooke, J. Acoust. Soc. Am. 101 (1997) 760.

[4] Y.Y. Lu, P.L. Ho, Opt. Lett. 27 (2002) 683.

[5] T. Anada, T. Hokazono, T. Hiraoka, J.P. Hsu, T.M. Benson, P. Sewell, IEICE Trans. Electron. E82-C (1999) 1154.

[6] G.R. Hadley, Opt. Lett. 17 (1992) 1426.

[7] S. Sujecki, J. Opt. Soc. Am. 25 (2007) 138.

[8] K. Kawano, T. Kitoh, Introduction to Optical Waveguide Analysis: Solving Maxwell's Equations and the Schrödinger Equation, John Wiley \& Sons, New York, 2001. p. 166.

[9] Y.Y. Lu, App. Numer. Math. 27 (1998) 141.

[10] G.R. Hadley, Opt. Lett. 17 (1992) 1743.

[11] C. Ma, E.V. Keuren, Opt. Express 14 (2006) 4668.

[12] C. Ma, E.V. Keuren, Opt. Express 15 (2007) 402.

[13] K.Q. Le, R.G. Rubio, P. Bienstman, G.R. Hadley, Opt. Express 16 (2008) 17021 